\title{
NOVEDADES PARA LA FLORA ARGENTINA ENCONTRADAS EN EL PARQUE NACIONAL IGUAZÚ (MISIONES)
}

\author{
por A. SCHININI ${ }^{1} \&$ M. GABRIELA LÓPEZ ${ }^{2}$
}

\section{Summary}

Two species are recorded for the first time for Argentina, Misiones: Solanum alternato-pinnatum Steud. (Solanaceae) known from Brasil and Paraguay and Aristolochia melastoma Silva Manso ex Duch. (Aristolochiaceae) before reported from Brasil.

Como parte del Inventario Florístico del Parque Nacional Iguazú (Misiones-Argentina), que se está realizando mediante un convenio entre la Facultad de Ciencias Agrarias (UNNE) y la Administración de Parques Nacionales, se dan a conocer dos nuevos taxones para la flora argentina, Solanum alternato-pinnatum Steud., especie que estaba registrada para Brasil y Paraguay y Aristolochia melastoma Silva Manso ex Duch. sólo de Brasil.

\section{Solanum alternato-pinnatumSteud.}

Fig. 1

Steudel, Nomencl. bot. 2: 600. 1841. Tipo: Velloso, Fl. flumin. Icones 2, lam. 125 (lectotipo!) 1831. Nuevo nombre para Solanum oleraceum Vell.

S. oleraceum Vell., Fl. flumin. Icones 2, lam. 125. 1831; Arq. Mus. Nac. Río de Janeiro 5: 84. 1881, non Dunal, 1813.

S. juciri Martius ex Sendtn., Martius, C., Fl. bras. 10: 110, 1846, «Mss. in itinerario Brasiliensi a. 1817. scripto n. 310». Chodat \& Vischer, Bull. Soc. Bot. Genève 2: 120-123, fig. 27, 1916, reprint 1977.

S. juciri f. paraguariense Hassler, Feddes Repert. 9: 118. 1911, «in silvis pr. Caaguazú, flor. mens. Mart.», Hassler 9365 (G!).

Planta perenne, con ramas de dos tipos, rastreras radicantes en los nudos, alcanzando ca. $12 \mathrm{~m}$ long. x $1 \mathrm{~cm}$ diám. y apoyantes,

\footnotetext{
${ }^{1}$ Instituto de Botánica del Nordeste.

${ }^{2}$ Facultad de Ciencias Agrarias. Instituto de Botánica del Nordeste, C.C. 209, 3400 Corrientes, Argentina.
}

trepadoras, 2-3 (-7) m long. x 0,5-5 mm diám., gráciles, verde claro, teretes, lisas en fresco, estriadas en seco, glabras, retrorso-aculeadas, acúleos hasta $2 \mathrm{~mm}$ long., de base ensanchada, aplanados, recurvos; lenticelas longitudinales, ca. 0,25 mm long., grisáceas en los bordes y centro castaño; entrenudos 2-5 (-8) $\mathrm{cm}$ long. Hojas alternas, pinnadas, papiráceas, planas; pecíolo de 1,5-5,5 cm long., retrorsoaculeado, al igual que el raquis, lámina 3,5-15 cm long., 3-7 folioladas, folíolos $1-10 \mathrm{~cm}$ long. $x$ 0,5-2,5 cm lat., el terminal de mayor tamaño, 4,5-11 cm long. x 1-4,5 cm lat., alternos $u$ opuestos, subsimétricos, desiguales, desde ovados a lanceolados, largamente atenuados hacia el ápice, base asimétrica, decurrente, epifilo verde intenso, hipofilo más claro, vena media retrorso-aculeada, principalmente en el hipofilo; algunos brotes axilares originan ramas muy cortas con hojas simples, atenuadas en un corto pecíolo de 0,2-0,7 cm long., lámina. $1-5 \mathrm{~cm}$ long. x 0,5-1,5 cm lat., ovada, levemente asimétrica, ápice agudo. Cimas corimbiformes, extra-axilares, péndulas, divaricadas, hasta de $28 \mathrm{~cm}$ long., ejes retrorso-aculeados; flores péndulas. Pedicelo, 5-15 mm long. x 0,30,5 $\mathrm{mm}$ lat. Cáliz campanulado, 4-6 $\mathrm{mm}$ long. x 5-8 mm lat., blanco-verdoso, 5-lobado, lóbulos redondeados, mucronados, 3-5,5 mm long. x 2-3 mm lat., glabros. Corola campanulada, 1,5-2 cm long. $x$ 0,7-1,8 cm lat., blanca, 5lobada, lóbulos ovados, 1,1-1,5 cm long. x 4-6 $\mathrm{mm}$ lat., glabros, ápice cuculado, apiculado, borde del extremo superior de los pétalos pu- 
bescente. Estambres 5, brevemente soldados entre sí y a la corola, el resto libre, filamentos desiguales, glabros, porción libre del más largo de 2,5-3 mm long. $x 1 \mathrm{~mm}$ lat., en los cuatro cortos muy breve de $0,25 \mathrm{~mm}$ long. $x 1 \mathrm{~mm}$ lat., anteras sub-basifijas, 5-7 mm long. x 1,5-2 $\mathrm{mm}$ lat., blancas, la dehiscencia comienza por un poro y continúa por una línea hacia la base, por lo tanto termina siendo longitudinal. Ovario ovoide, 1,5-2 mm long. x 1,5 mm lat., estilo simple, algo engrosado en el extremo, de 7-9 $\mathrm{mm}$ long., glabro. Baya esférica, de 3-5 cm diám., péndula, pedicelo 1,5-2 cm long., con el cáliz persistente, lisa, verde-olivácea, con áreas irregulares blanquecinas que se unen en el ápice, cuando madura anaranjada y el cáliz reflexo. Semillas orbiculares, 4-5 mm diám.

Material examinado: ARGENTINA. Misiones. Dep. Iguazú, Parque Nacional Iguazú, Ruta 101, 3III-1982, Ferraro 2441 (CTES). Sendero Jacaratia, 16VI-1995, Malmierca \& al. 56 (CTES). Ruta 101, estación «Palo Rosa» $5 \mathrm{~km}$ límite E del Parque, Puesto Yacuí, 3-IV-1997, Vanni, López, Kurtz y Herrera 3950 (CORD, CTES, G, MBM, US). Dep. Cainguás, Ruta 7,5 Km W de acceso a Aristóbulo del Valle, 30-VII-1987, Vanni \& al. 886 (CTES, GH, K, LIL).

BRASIL. Paraná. Cerro Azul, Cab. Rib. do Tigre, 24-IV-1987, Hatschbach \& al. 51229 (CTES). Idem, 23-XI-1979, Hatschbach 42593 (CTES).

PARAGUAY. Alto Paraná. Reserva Biológica Itabó, 19-III-1982, Caballero Marmori s/n (CTES, HERB. ITAIPÚ-973). Reserva Biológica Limoy, 10VI-1981, Caballero Marmori s/n (CTES, HERB. ITAIPÚ-986). Guairá. Colonia Independencia, III1924, Rojas 4868 (AS, CTES). Cordillera. Cordillera de Altos, Loma Porá, 4-XII-1949, Rojas 10733 (AS, CTES).

Distribución geográfica y habitat: vive en el centro y SE de Brasil en los Estados de Goiás, Río de Janeiro, San Pablo, Paraná y Santa Catarina; E de Paraguay y NE de Argentina, donde habita en los márgenes e interior de selva.

Obs. 1. S. alternato-pinnatum pertenece al subgénero Leptostemonum (Dunal) Bitter, sección Aculeigerum Seithe, subsección Juciri Marzell (D'Arcy, 1972: 275). Whalen (1984: 208) la ubica en el grupo Solanum wendlandii, que incluye lianas glabras o con pocos y diminutos pelos simples o ramificados, no estrellados y pequeños aguijones recurvados, con los cuales pueden sostenerse, en tailo, pecíolo y vena media del hipofilo. Esta especie es afín a $S$. wendlandii Hook. f., nativa de Costa Rica y ampliamente cultivada en regiones subtropicales y templadas (Smith \& Downs, 1966: 140). Se cultiva también en Paraguay y Argentina.

Obs. 2. Entre las especies de Solanum citadas para Argentina (Morton, 1976) es afín a S. sisymbriifolium Lam. (Secc. Pseudocryptocarpum Marzell = Secc. Cryptocarpum Dunal). Esta especie es anual o bienal, con hojas pinnatífidas o bipinnatífidas, cáliz espinoso, acrescente encerrando al fruto; S. alternato-pinnatum en cambio, es perenne, posee hojas pinnadas y cáliz liso no acrescente.

\section{Aristolochia melastoma Silva Manso ex Duch.}

Fig. 2

Duchartre, en DC. Prodr. 15 (1): 460-461, 1864. Masters en Martius, Fl. bras. 4 (2): 103, tab. 22, fig. 1, 1875. Hoehne, Mem. Inst. Oswaldo Cruz 20 (1): 74 , tab. 70, fig. 1, 1927. Ahumada, Aristoloquiáceas en Reitz, Flora Ilustrada Catarinense, Fasc. ARIS: 32, fig. 10, 1975.

Fanerófita escandente con ramas de dos tipos, unas rastreras con nudos engrosados, radicantes, otras volubles de hasta $4 \mathrm{~m}$ alt., estriadas, pubescentes, pelos de 0,25-0,5 mm long., más abundantes en partes jóvenes, pluricelulares, incurvos, base ensanchada, hialinos; entrenudos (5-) 6-12 (-13) cm long. Hojas pecioladas sin pseudoestípulas. Pecíolo de 0,5-3,5 cm long., estriado en seco, canaliculado en la cara superior en fresco, pubescente. Lámina de (3,5-) 6-12 (-14) cm long. x (1,3-) 3-5 cm lat., lanceolada o aovada, subsimétrica, papirácea, ápice atenuado, levemente arqueado, base cordada, superficie rugoso-papilosa, 3 venas basales, venas secundarias pinnadas, anastomosadas entre sí hacia el borde; margen entero, ligeramente sinuoso; epifilo verde-oliváceo, pubérulo, pelos adpresos, hipofilo verde-claro, con pelos cortos, adpresos; venas sobresalientes más claras, con 


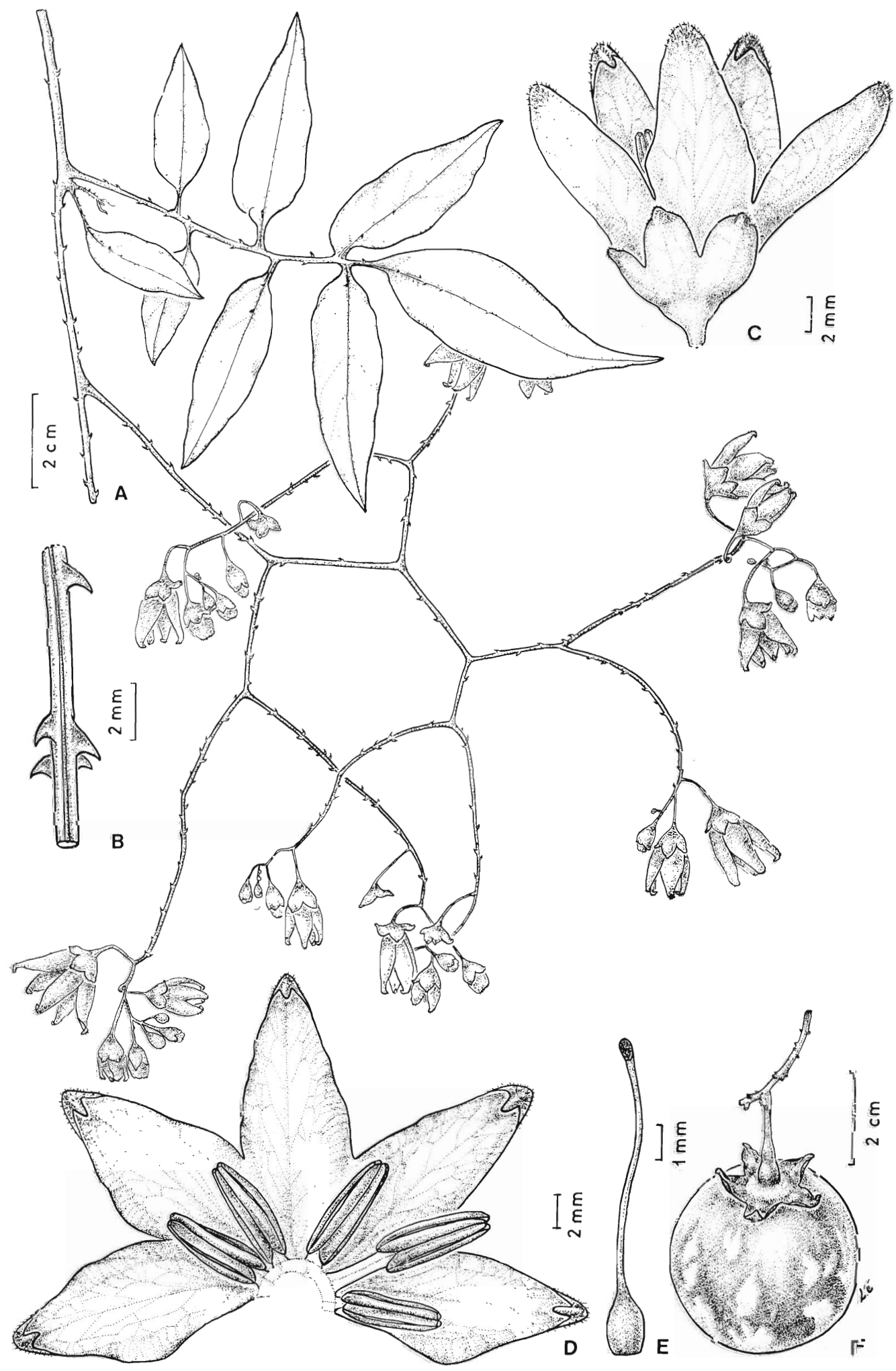

Fig.1. Solanum alternato-pinnatum: A, rama florífera, hoja (hipofilo); B, detalle del tallo; C, flor, vista lateraī; D, flor abierta; E, gineceo; F, fruto. (Vanni \& al. 3950, CTES). 
pelos largos y densamente dispuestos. Racimos axilares, depauperados, pubescentes, a veces 2-3 fasciculados, 1-4 cm long., 2-7-floros, brácteas opuestas a las flores de 1,5-6 mm long., aovadas, pubescentes. Pedicelo y ovario 5-10 mm long., pubescentes, perpendiculares al eje del racimo. Perigonio unilabiado de 2$4,5 \mathrm{~cm}$ long., externamente pubescente, pubescencia más densa en las venas, pelos pluricelulares de dos tipos unos cortos con 4-7 células y otros largos con 9-11, la célula basal estriada y la apical uncinulada, que se desprende al estrangularse la célula anterior; utrículo 5-10 mm long. x 4-8 mm lat., elipsoidal, blanco, cara interna cubierta de pelos, largos, finos, entrelazados, dispuestos en manojos, de paredes delgadas, de 0,75-0,85 mm long. x 0,01 mm lat., formados por 30-37 células; en el lado dorsal del utrículo mancha de pelos densamente dispuestos, largos, finos, con paredes engrosadas, de 0,25-0,52 mm long. $x$ 0,014 mm lat., rodeada por pelos cortos, gruesos, con paredes delgadas, de 0,18-0,2 mm long. $x \quad 0,02-0,025 \mathrm{~mm}$ lat., formados por 11-15 células; tubo castaño-oscuro hasta las fauces, cilíndrico, lado ventral giboso en la base, el dorsal cóncavo; la porción inferior interna, que penetra en la cavidad utricular, subcilíndrica, glabra, lisa; labio expandido rodeando las fauces, lado inferior angosto en forma de reborde, emarginado en el centro, lado superior ovado, $1-3 \mathrm{~cm}$ long. x 1-2 cm lat., 5 nervado, cara externa castaño-oscura, cara interna dividida en dos áreas: una lisa, próxima a las fauces formando un arco amarillo-oro, color que se extiende por las venas hacia el extremo del labio, con pelos pediculados, retrorsos, pluricelulares, 0,5-1,5 mm long., dispersos también en la cara interna del tubo; la otra área en el tercio superior del labio, castaño-oscura, papilosa y con verrugas aisladas atropurpúreas. Ginostemio pediculado, subcilíndrico, 2,5-4 $\mathrm{mm}$ long., pedículo $0,5-1,25 \mathrm{~mm}$ long. $x$ 0,25-0,5 mm lat., anteras 6 , 1-1,2 mm long., oblongas, dispuestas por debajo de los lóbulos estigmatíferos. Lóbulos 6, libres en la mitad superior, subtriangulares, con los bordes papilosos, terminados en un apéndice.
Material examinado: ARGENTINA. Misiones. Dep. Iguazú, Parque Nacional Iguazú. Limite E del Parque, a $1000 \mathrm{~m}$ de la costa del río Iguazú, 1-III1996, Vanni \& al. 3559 (CTES, MBM, SI).

BRASIL. Minas Gerais. Sete Lagoas, 13-II-1973, Hatschbach \& Ahumada 31513 (MBM); Pedro Leopoldo, 12-II-1973, Hatschbach \& Ahumada 31485 (MBM); mun. Caeté, Caeté, 12-II-1973, Hatschbach \& Ahumada 31473 (MBM); mun. Caparaó, Caparaó Novo, 16-II-1973, Hatschbach \& Ahumada 31462 (MBM); mun. Caldas, Caldas, 27-I1860, Regnell III-1043 (SP). São Paulo. Campinas, Campos Novaes 935 (SP); São Paulo, «Nativa no Jardim Botânico, planta viva $n^{\circ}$ 428», 20-II-1936, Hoehne s/n (SP 32300); Butantan, III-1918, Hoehne $\mathrm{s} / \mathrm{n}(\mathrm{SP} 1470)$.

Distribución geográfica y hábitat: vive en Brasil, en los Estados de Minas Gerais, Río de Janeiro, São Paulo y Paraná, donde habita en bordes de selva y campos con suelo removido. En Misiones, Argentina, fue coleccionada en una picada en la selva.

Obs. 1. En cada inflorescencia se presenta una sola flor en antesis, en algunas se pueden ver distintos estados de desarrollo floral, botón incipiente, flor en antesis y fruto en formación, Fig. 2. A.

Obs. 2. De acuerdo con Hoehne (1942: 26) A. melastoma pertenece al grupo Extipulatae por carecer de estípulas; la especie más próxima es A. acutifolia Duch. que vive desde Amazonas hasta Ceará. Se diferencian en que $A$. acutifolia carece de verrugas en el labio del perigonio y tiene hojas cartáceas ${ }^{3}$ mientras que $A$. melastoma posee verrugas y hojas papiráceas.

\section{Agradecimientos}

Queremos expresar nuestro agradecimiento a la Prof. Rosa Guaglianone y al Señor Justo Herrera becario del Parque Nacional Iguazú por la colaboración que nos han brindado, a la Ing. Agr. Ana María González por la ayuda

\footnotetext{
${ }^{3}$ De A. acutifolia se estudió el ejemplar. BRASIL. Pará, Belem, Canudos, 9-1914, s/c. (SP 17796).
} 


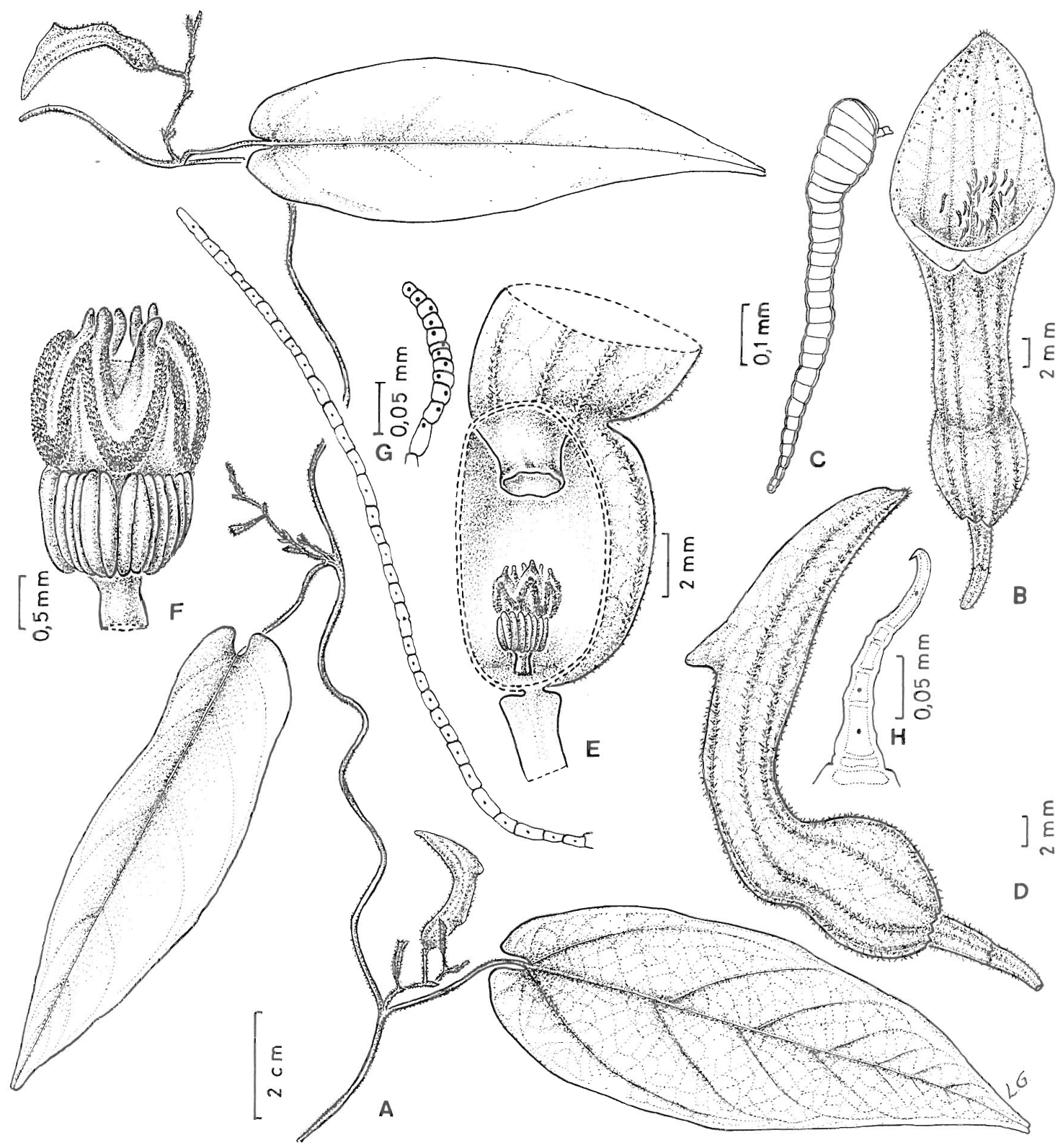

Fig. 2. Aristolochia melastoma: A, rama florífera; $B$, flor vista de frente; $C$, detalle de un pelo pediculado de las fauces; D, flor en vista lateral; E, corte longitudinal del utrículo, no se dibujó el indumento de la cara interna; F, ginostemio; $\mathrm{G}$, pelos del interior del utrículo, el largo tomado de un manojo, el corto del borde de la mancha; $\mathrm{H}$, pelo de la cara externa del perigonio. (Vanni \& al. 3559, CTES, B-H de material conservado en AFA). 
brindada en la interpretación de los pelos y a la Sra. Liliana Gómez por la realización de las láminas.

\section{Bibliografía}

D'ARCY, Z.G., 1972. Solanaceae Studies II: Typification of Subdivisions of Solanum, Ann. Missouri Bot. Gard. 59: 262-278.

DUNAL, M.F., 1852. Solanaceae en A.P. De Candolle, Prodr.
13 (1): 50-51. París.

HOEHNE, F. C., 1942. Aristolochiaceas. Flora Brasílica 15 (2): 91-92, tab. 61.

MORTON, C.V., 1976. A Revision of the Argentine Species of Solanum, págs. 1-260, f. 1-30. Academia Nacional de Ciencias. Córdoba.

SMITH, L.B. \& R.J. DOWNS, 1966. Solanáceas en P.R. Reitz, Flora Ilustrada Catarinense, Fasc. SOLA: 139-140.

WHALEN, D.W. 1984. Conspectus of Species Groups in Solanum Subgenus Leptostemonum. Gentes Herbarum 12 (4): 179-282. 